\title{
Structural basis for the inhibition of SARS-CoV-2 main protease by antineoplastic drug carmofur
}

\author{
Zhenming Jin $\odot^{1,2,8}$, Yao Zhao ${ }^{1,8}$, Yuan Sun ${ }^{3,8}$, Bing Zhang ${ }^{1}$, Haofeng Wang ${ }^{1,4}$, Yan Wu ${ }^{3}$, Yan Zhu', \\ Chen Zhu', Tianyu Hu', Xiaoyu Du ${ }^{1,2}$, Yinkai Duan', Jing Yu', Xiaobao Yang', Xiuna Yang1, \\ Kailin Yang ${ }^{5}$, Xiang Liu $\oplus^{6}$, Luke W. Guddat ${ }^{7}$, Gengfu Xiao ${ }^{3}$, Leike Zhang $\oplus^{3 凶}{ }^{\circledR}$, Haitao Yang $\oplus^{1 凶}$ \\ and Zihe Rao ${ }^{1,2,6}$
}

\begin{abstract}
The antineoplastic drug carmofur is shown to inhibit the SARS-CoV-2 main protease $\left(M^{\text {pro }}\right)$. Here, the X-ray crystal structure of $M^{\text {pro }}$ in complex with carmofur reveals that the carbonyl reactive group of carmofur is covalently bound to catalytic Cys145, whereas its fatty acid tail occupies the hydrophobic S2 subsite. Carmofur inhibits viral replication in cells $\left(E_{50}=24.30 \mu \mathrm{M}\right)$ and is a promising lead compound to develop new antiviral treatment for COVID-19.
\end{abstract}

COVID-19, a highly infectious viral disease caused by coronavirus SARS-CoV-2, has spread worldwide since its appearance in December 2019, causing an unprecedented pandemic. The number of confirmed cases worldwide continues to grow at a rapid rate, but, at this time, there are no specific drugs or vaccines available to control the symptoms or the spread of this disease.

SARS-CoV-2 has an $\sim 30,000$ nt RNA genome. The first open reading frame encodes two translational products, polyproteins $1 \mathrm{a}$ and $1 \mathrm{ab}$ (ppla and pplab) ${ }^{1,2}$, which are processed into mature non-structural proteins by the main protease $\left(\mathrm{M}^{\mathrm{pro}}\right)$ and a papain-like protease ${ }^{3} . \mathrm{M}^{\text {pro }}$ has been proposed as a therapeutic target for anti-coronavirus drug development ${ }^{4-6}$. We previously screened over 10,000 compounds and identified carmofur as a compound that can inhibit $\mathrm{M}^{\text {pro }}$ in vitro, with a half-maximum inhibitory concentration $\left(\mathrm{IC}_{50}\right)$ of $1.82 \mu \mathrm{M}$ (ref. ${ }^{7}$ ).

Carmofur (1-hexylcarbamoyl-5-fluorouracil) is a derivative of 5-fluorouracil (5-FU; Fig. 1a) and an approved antineoplastic agent. Carmofur has been used to treat colorectal cancer since the $1980 \mathrm{~s}^{8}$ and has shown clinical benefits for breast, gastric and bladder cancers ${ }^{9-11}$. The target for carmofur is believed to be thymidylate synthase ${ }^{12,13}$, but it has also been shown to inhibit human acid ceramidase $(\mathrm{AC})^{14}$ through covalent modification of its catalytic cysteine ${ }^{15}$.

The molecular details of how carmofur inhibits $\mathrm{M}^{\text {pro }}$ activity have remained unresolved. Here, we now present a $1.6 \AA$ X-ray crystal structure of SARS-CoV-2 $\mathrm{M}^{\text {pro }}$ in complex with carmofur (Fig. 1b,c and Supplementary Table 1). In agreement with previous studies ${ }^{4,5,16-18}, \mathrm{M}^{\text {pro }}$ forms a homodimer (protomers A and B) related by crystallographic symmetry (Extended Data Fig. 1a,b). All of the residues (1-306) in the polypeptide can be traced in the electron density map. Each protomer is composed of three domains (Fig. 1c) - domains I (residues 10-99), II (residues 100-184) and III (residues 201-303) — and a long loop region (residues 185-200) that connects domains II and III. The substrate-binding pocket lies in the cleft between domains I and II, and features the catalytic dyad residues Cys145 and His41 (Fig. 1c,d). The substrate-binding pocket is divided into a series of subsites (including S1, S2, S4 and S1'), each accommodating a single but consecutive amino acid residue in the substrate. Ser1 in each one protomer interacts with Phe140 and Glu166 of the other protomer to stabilize the S1 subsite (Extended Data Fig. 1c), a structural feature that is essential for catalysis?

The electron density map unambiguously shows that the fatty acid moiety $\left(\mathrm{C}_{7} \mathrm{H}_{14} \mathrm{NO}\right)$ of carmofur is linked to the $\mathrm{S} \gamma$ atom of Cys 145 through a $1.8 \AA$ covalent bond, whereas the fatty acid tail is inserted into the S2 subsite (Fig. 1d,e). This observation suggests that the sulfhydryl group of Cys145 attacks the electrophilic carbonyl group of carmofur, resulting in covalent modification of Cys145 and release of the 5-FU moiety (Fig. $1 \mathrm{~b}$ and Extended Data Fig. 2a). In addition to the C-S covalent bond, the inhibitor is stabilized by numerous hydrogen bonds and hydrophobic interactions (Fig. 1e and Extended Data Fig. 2b). The carbonyl oxygen of carmofur occupies the oxyanion hole and forms hydrogen bonds $(3.0 \AA)$ with the backbone amides of Gly143 and Cys145, mimicking the tetrahedral oxyanion intermediate formed during protease cleavage (Fig. 1e). The fatty acid tail, which appears in an extended conformation, inserts into the bulky hydrophobic S2 subsite (composed of the side chains of His41, Met49, Tyr54 and Met165 and the alkyl portion of the side chain of Asp187; Fig. 1d,e). The hydrophobic interactions are mainly contributed by the side chains of His41, Met49 and Met165, all of which run parallel to the alkyl part of the fatty acid tail of the inhibitor (Fig. 1e and Extended Data Fig. 2b).

The mechanism of covalent modification by carmofur is different from that of the inhibitor $\mathrm{N}^{7,19}$, which covalently modifies Cys145 through Michael addition of the vinyl group. The structures of $\mathrm{M}^{\text {pro }}$-carmofur and $\mathrm{M}^{\text {pro }}-\mathrm{N} 3$ are similar overall (r.m.s. deviation (r.m.s.d.) of $0.286 \AA$ for all $\mathrm{C} \alpha$ atoms). The largest conformational differences occur in the substrate-binding pocket, with the backbone surrounding carmofur in a slightly more outward position

\footnotetext{
'Shanghai Institute for Advanced Immunochemical Studies and School of Life Science and Technology, ShanghaiTech University, Shanghai, China. 2Laboratory of Structural Biology, School of Life Sciences and School of Medicine, Tsinghua University, Beijing, China. ${ }^{3}$ State Key Laboratory of Virology, Wuhan Institute of Virology, Center for Biosafety Mega-Science, Chinese Academy of Sciences, Wuhan, China. ${ }^{4}$ School of Life Sciences, Tianjin University, Tianjin, China. ${ }^{5}$ Taussig Cancer Center, Cleveland Clinic, Cleveland, OH, USA. ${ }^{6}$ State Key Laboratory of Medicinal Chemical Biology, Frontiers Science Center for Cell Response, College of Life Sciences, College of Pharmacy, Nankai University, Tianjin, China. ${ }^{7}$ School of Chemistry and Molecular Biosciences, the University of Queensland, Brisbane, Australia. ${ }^{8}$ These authors contributed equally: Zhenming Jin, Yao Zhao, Yuan Sun. ${ }^{凶} \mathrm{e}-\mathrm{mail}$ : zhangleike@wh.iov.cn; yanght@shanghaitech.edu.cn
} 
<smiles>CCCCCCNC(=O)n1c(C)c(F)c(=O)[nH]c1=O</smiles><smiles>CCCCCCNC(=O)SCCCCC</smiles>

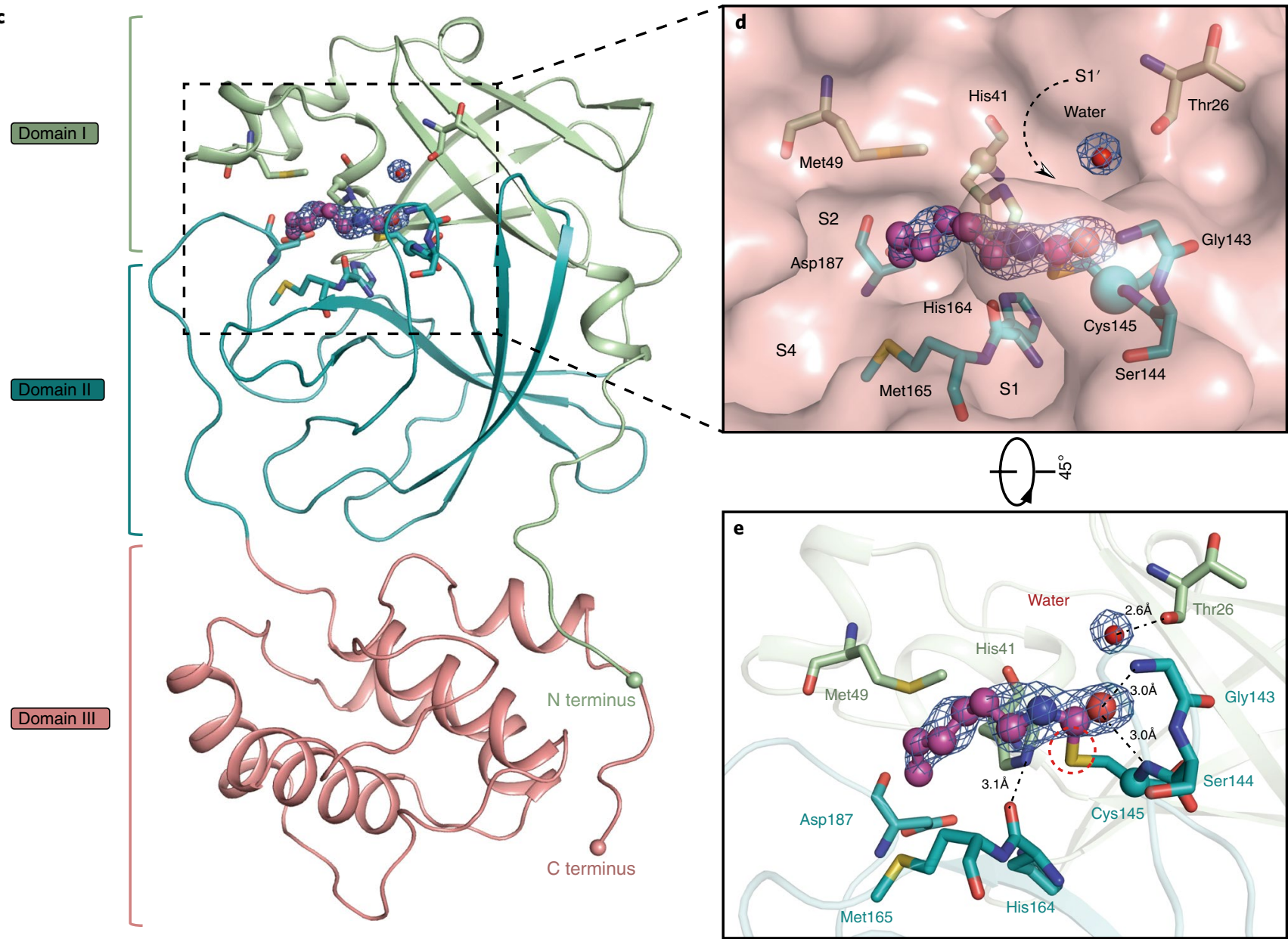

Fig. 1 | SARS-CoV-2 $\mathbf{M}^{\text {pro }}$ in complex with carmofur. a, The chemical structure of carmofur. $\mathbf{b}$, The binding mode of carmofur to SARS-CoV-2 Mro. The red curve represents the SARS-CoV- $2 \mathrm{M}^{\text {pro }}$ polypeptide with the side chain of Cys 145 protruding. c, The structure of a single protomer. The three domains are shown in three different colors. The catalytic center is located within the dashed square. d, Magnified view of the catalytic center. The residues that participate in carmofur binding are shown as stick models. Carmofur is shown as a ball-and-stick model with the carbons in magenta. A water molecule is presented as a red sphere. e, A rotated view of the binding site, but with the surface removed. The red dashed circle highlights the $\mathrm{C}-\mathrm{S}$ covalent bond.

compared with the $\mathrm{M}^{\text {pro-N}}$ 3 complex structure (Extended Data Fig. 3a). Another difference is that carmofur only occupies the S2 subsite (Fig. 1d), whereas N3 occupies four subsites (S1, S2, S4 and $\mathrm{S1}^{\prime}$; Extended Data Fig. 3b,c). The lactam ring of N3 is located in the S1 subsite, which is filled by a DMSO molecule in the $\mathrm{M}^{\text {pro-carmo- }}$ fur structure (Extended Data Fig. 3b,c). These observations demonstrate the potential for structural elaboration of carmofur and will be useful for the design of more potent derivatives against the $\mathrm{M}^{\text {pro }}$ of SARS-CoV-2.

We previously showed that treatment with $10 \mu \mathrm{M}$ ebselen (half-maximum effective concentration, $\mathrm{EC}_{50}=4.67 \mu \mathrm{M}$ ) inhibited infection of Vero cells with SARS-CoV-2, whereas carmofur did not show detectable antiviral activity at this concentration ${ }^{7}$. Here, we have determined the inhibitory effect of carmofur against SARS-CoV-2 infection on Vero E6 cells, as previously described in ref. ${ }^{20}$ (Fig. 2). By measuring viral RNA in the cellular supernatant, we determined the $\mathrm{EC}_{50}$ value for carmofur as $24.30 \mu \mathrm{M}$ (Fig. 2a). To verify this result, we fixed infected cells and stained them using anti-sera against viral nucleocapsid protein (NP), observing a decrease in NP levels after carmofur treatment (Fig. 2b). We also performed cytotoxicity assays for carmofur in Vero E6 cells and determined a half-maximum cytotoxic concentration $\left(\mathrm{CC}_{50}\right)$ value of $133.4 \mu \mathrm{M}$ (Fig. 2c). Thus, carmofur has a favorable selectivity index (SI) of 5.36, but further optimization will be required to develop an effective drug.

In conclusion, the crystal structure of $\mathrm{M}^{\text {pro }}$ in complex with carmofur shows that the compound directly modifies the catalytic Cys145 of SARS-CoV-2 $\mathrm{M}^{\text {pro }}$. Our study also provides a basis for rational design of carmofur analogs with enhanced inhibitory efficacy to treat COVID-19. Because $\mathrm{M}^{\text {pro }}$ is highly conserved among all 

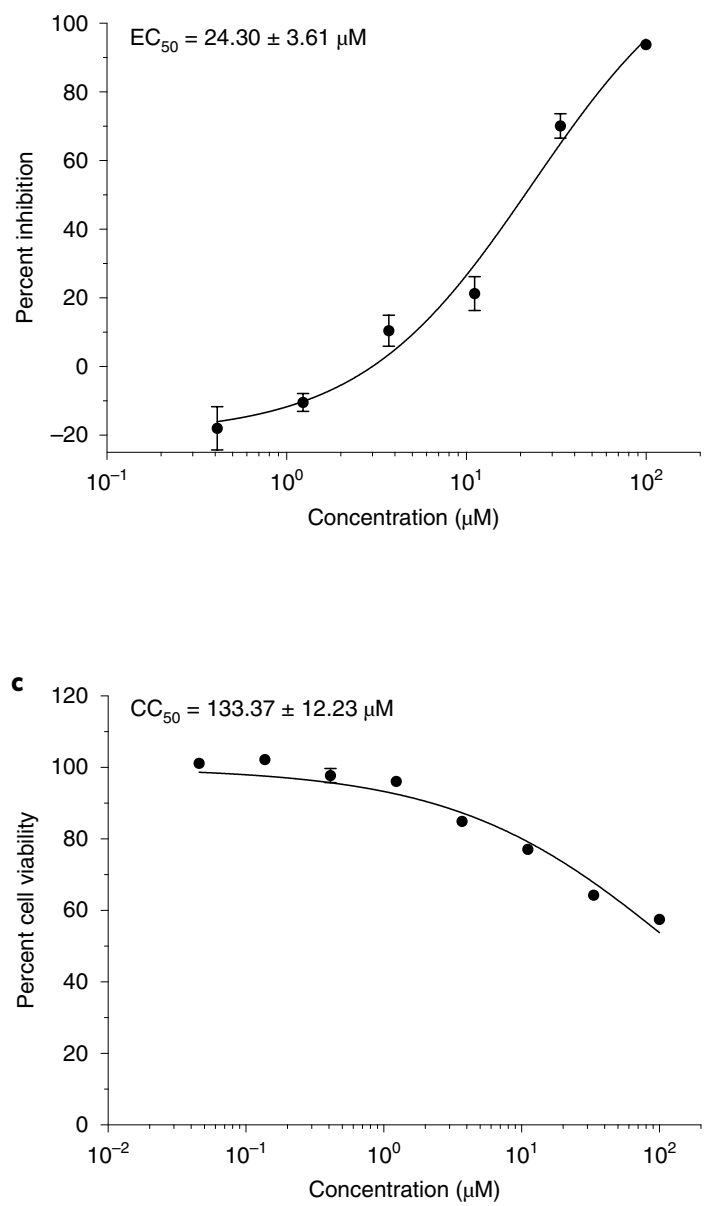

b
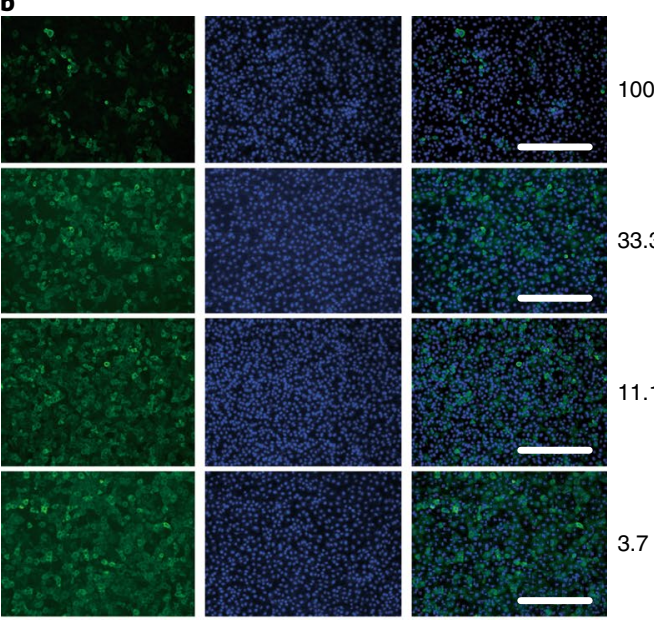

33.3
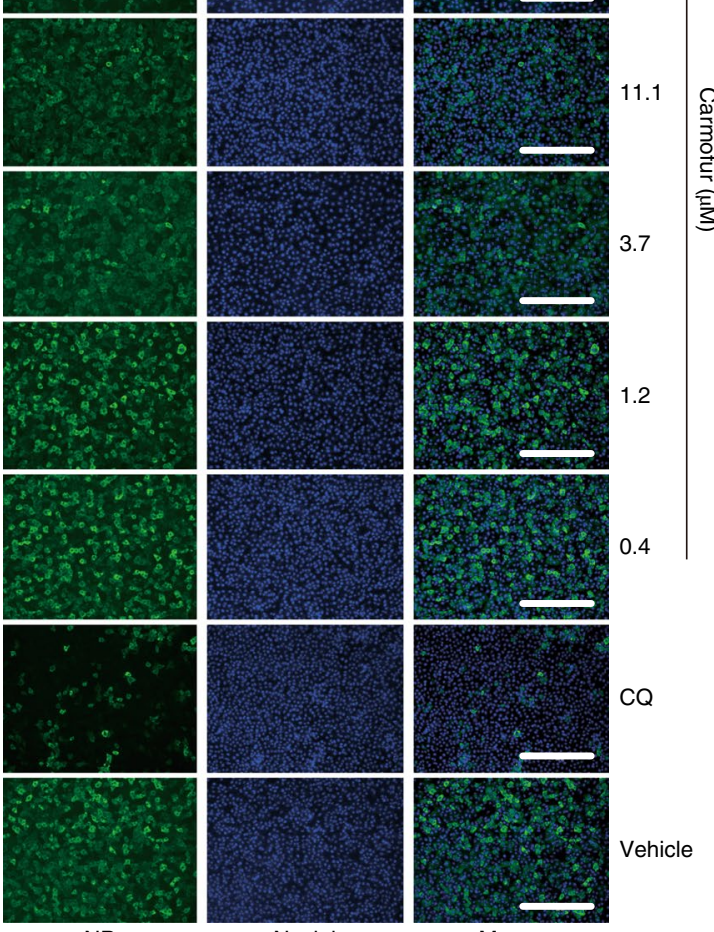

Nuclei

Fig. 2 | Inhibition of SARS-CoV-2 by carmofur in Vero E6 cells. Vero E6 cells infected with SARS-CoV-2 at a multiplicity of infection (MOI) of 0.05 were treated with different concentrations of carmofur. a, Quantitative polymerase chain reaction with reverse transcription (qRT-PCR) assays were performed to measure the viral copy number in cellular supernatant. The $y$ axis indicates percentage inhibition of virus relative to sample treated with DMSO (vehicle). Data are shown as mean \pm s.e.m., $n=6$ biological replicates. $\mathbf{b}$, Immunofluorescence images for intracellular NP. At $24 \mathrm{~h}$ post-infection, cells were fixed, and intracellular NP levels were monitored by immunofluorescence. Chloroquine $(C Q, 10 \mu \mathrm{M})$ was used as a positive control ${ }^{20}$. The results are representative of three biological replicates. Scale bars, $400 \mu \mathrm{m}$. c, Cell viability assay. The $y$ axis represents the percentage of cell viability relative to sample treated with DMSO (vehicle). Data are shown as mean \pm s.e.m., $n=3$ biological replicates. Data for graphs in $\mathbf{a}$ and $\mathbf{c}$ are available as source data.

coronaviruses, carmofur and its analogs may be effective against a broader spectrum of these viruses.

\section{Online content}

Any methods, additional references, Nature Research reporting summaries, source data, extended data, supplementary information, acknowledgements, peer review information; details of author contributions and competing interests; and statements of data and code availability are available at https://doi.org/10.1038/s41594020-0440-6.

Received: 8 April 2020; Accepted: 27 April 2020;

Published online: 7 May 2020

\section{References}

1. Wu, F. et al. A new coronavirus associated with human respiratory disease in China. Nature 579, 265-269 (2020).

2. Zhou, P. et al. A pneumonia outbreak associated with a new coronavirus of probable bat origin. Nature 579, 270-273 (2020).

3. Hegyi, A. \& Ziebuhr, J. Conservation of substrate specificities among coronavirus main proteases. J. Gen. Virol. 83, 595-599 (2002).
4. Anand, K. et al. Structure of coronavirus main proteinase reveals combination of a chymotrypsin fold with an extra $\alpha$-helical domain. EMBO J. 21, 3213-3224 (2002).

5. Yang, $\mathrm{H}$. et al. The crystal structures of severe acute respiratory syndrome virus main protease and its complex with an inhibitor. Proc. Natl Acad. Sci. USA 100, 13190-13195 (2003)

6. Pillaiyar, T., Manickam, M., Namasivayam, V., Hayashi, Y. \& Jung, S. H. An overview of severe acute respiratory syndrome-coronavirus (SARS-CoV) 3CL protease inhibitors: peptidomimetics and small molecule chemotherapy. J. Med. Chem. 59, 6595-6628 (2016).

7. Jin, Z. et al. Structure of $\mathrm{M}^{\text {pro }}$ from COVID-19 virus and discovery of its inhibitors. Nature https://doi.org/10.1038/s41586-020-2223-y (2020).

8. Sakamoto, J. et al. An individual patient data meta-analysis of adjuvant therapy with carmofur in patients with curatively resected colon cancer. Jpn J. Clin. Oncol. 35, 536-544 (2005).

9. Morimoto, K. \& Koh, M. Postoperative adjuvant use of carmofur for early breast cancer. Osaka City Med. J. 49, 77-83 (2003).

10. Gröhn, P. et al. Oral carmofur in advanced gastrointestinal cancer. Am. J. Clin. Oncol. 13, 477-479 (1990).

11. Nishio, S. et al. Study on effectiveness of carmofur (Mifurol) in urogenital carcinoma, especially bladder cancer, as a post-operative adjuvant chemotherapeutic agent. Hinyokika Kiyo 33, 295-303 (1987). 
12. Ooi, A. et al. Plasma, intestine and tumor levels of 5-fluorouracil in mice bearing L1210 ascites tumor following oral administration of 5-fluorouracil, UFT (mixed compound of tegafur and uracil), carmofur and 5'-deoxy5-fluorouridine. Biol. Pharm. Bull. 24, 1329-1331 (2001).

13. Sato, S., Ueyama, T., Fukui, H., Miyazaki, K. \& Kuwano, M. Anti-tumor effects of carmofur on human 5-FU resistant cells. Gan To Kagaku Ryoho 26, 1613-1616 (1999).

14. Nguyen, H. S., Awad, A. J., Shabani, S. \& Doan, N. Molecular targeting of acid ceramidase in glioblastoma: a review of its role, potential treatment and challenges. Pharmaceutics 10, E45 (2018).

15. Dementiev, A. et al. Molecular mechanism of inhibition of acid ceramidase by carmofur. J. Med. Chem. 62, 987-992 (2019).

16. Xue, X. et al. Structures of two coronavirus main proteases: implications for substrate binding and antiviral drug design. J. Virol. 82, 2515-2527 (2008).
17. Ren, Z. et al. The newly emerged SARS-like coronavirus HCoV-EMC also has an 'Achilles' heel': current effective inhibitor targeting a 3C-like protease. Protein Cell 4, 248-250 (2013).

18. Wang, F., Chen, C., Tan, W., Yang, K. \& Yang, H. Structure of main protease from human coronavirus NL63: insights for wide spectrum anti-coronavirus drug design. Sci. Rep. 6, 22677 (2016).

19. Yang, H. et al. Design of wide-spectrum inhibitors targeting coronavirus main proteases. PLoS Biol. 3, e324 (2005).

20. Wang, M. et al. Remdesivir and chloroquine effectively inhibit the recently emerged novel coronavirus (2019-nCoV) in vitro. Cell Res. 30, 269-271 (2020).

Publisher's note Springer Nature remains neutral with regard to jurisdictional claims in published maps and institutional affiliations.

(c) The Author(s), under exclusive licence to Springer Nature America, Inc. 2020 


\section{Methods}

Cloning, protein expression and purification of SARS-CoV-2 $\mathrm{M}^{\text {pro }}$. The cell cultures were grown and the protein expressed according to the method in ref. 7 . The cell pellets were resuspended in lysis buffer $(20 \mathrm{mM}$ Tris-HCl pH 8.0, $150 \mathrm{mM}$ $\mathrm{NaCl}, 5 \%$ glycerol), lysed by high-pressure homogenization and then centrifuged at $25,000 \mathrm{~g}$ for $30 \mathrm{~min}$. The supernatant was loaded onto a Ni-NTA affinity column (Qiagen) and washed in lysis buffer containing $20 \mathrm{mM}$ imidazole. The His-tagged $\mathrm{M}^{\text {pro }}$ was eluted by lysis buffer that included $300 \mathrm{mM}$ imidazole. The imidazole was then removed through desalting. Human rhinovirus $3 \mathrm{C}$ protease was added to remove the $\mathrm{C}$-terminal His tag. The SARS-CoV-2 $\mathrm{M}^{\text {pro }}$ was further purified by ion exchange chromatography, then the purified $\mathrm{M}^{\mathrm{pro}}$ was transferred to $10 \mathrm{mM}$ Tris- $\mathrm{HCl} \mathrm{pH} 8.0$ by desalting and stored at $-80^{\circ} \mathrm{C}$ until needed.

Crystallization, data collection and structure determination. SARS-CoV- $2 \mathrm{M}^{\text {pro }}$ was concentrated to $5 \mathrm{mg} \mathrm{ml}^{-1}$, incubated with $0.3 \mathrm{mM}$ carmofur (Selleck) for $1 \mathrm{~h}$, then the complex was crystallized by the hanging drop vapor diffusion method at $20^{\circ} \mathrm{C}$. The best crystals were grown using a well buffer containing $0.1 \mathrm{M}$ MES pH 6.0, 5\% polyethylene glycol 6000 and 3\% DMSO. The cryo-protectant solution was the reservoir but with $20 \%$ glycerol added.

X-ray data were collected on beamline BL17U1 at Shanghai Synchrotron Radiation Facility (SSRF) at $100 \mathrm{~K}$ and at a wavelength of $0.97918 \AA$ using an Eiger $\mathrm{X} 16 \mathrm{M}$ image plate detector. Data integration and scaling were performed using the program $\mathrm{XDS}^{21}$. The structure was determined by molecular replacement (MR) with PHASER ${ }^{22}$ and Phenix 1.17.1.13 using SARS-CoV-2 M M $^{\text {pro }}$ (PDB ID 6LU7) as a search template. The model from MR was subsequently subjected to iterative cycles of manual model adjustment with Coot $0.8^{24}$, and refinement was completed with Phenix REFINE ${ }^{25}$. The inhibitor, carmofur, was built according to the omit map. The phasing and refinement statistics are summarized in Supplementary Table 1.

Antiviral and cytotoxicity assays for carmofur. A clinical isolate of SARS-CoV-2 (nCoV-2019BetaCoV/Wuhan/WIV04/2019) was propagated in Vero E6 cells, and the viral titer was determined as described previously ${ }^{20}$. Vero E6 cells were obtained from ATCC with authentication. Authentication was performed by a morphology check under a microscope and growth curve analysis. All cells were tested as mycoplasma-negative. For the antiviral assay, pre-seeded Vero E6 cells $\left(5 \times 10^{4}\right.$ cells per well) were pre-treated with different concentrations of carmofur for $1 \mathrm{~h}$ and the virus was subsequently added (MOI of 0.05 ) to allow infection for $1 \mathrm{~h}$. Next, the virus-drug mixture was removed and the cells were further cultured with fresh drug-containing medium. At $24 \mathrm{~h}$ post-infection, the cell supernatant was collected and vRNA in supernatant was subjected to qRT-PCR analysis, while cells were fixed and subjected to immunofluorescence to monitor intracellular NP level as described previously ${ }^{20}$. For cytotoxicity assays, Vero E6 cells were suspended in growth medium in 96-well plates. The next day, appropriate concentrations of carmofur were added to the medium. After $24 \mathrm{~h}$, the relative numbers of surviving cells were measured using a Cell Counting Kit- 8 (CCK8, Beyotime) assay in accordance with the manufacturer's instructions. All experiments were performed in triplicate, and all the infection experiments were performed at biosafety level 3 (BSL-3).

Reporting Summary. Further information on research design is available in the Nature Research Reporting Summary linked to this article.

\section{Data availability}

Coordinates and structure factors for SARS-CoV- $2 \mathrm{M}^{\text {pro }}$ in complex with carmofur have been deposited in the Protein Data Bank (PDB) under accession code 7BUY. Source data for Fig. 2 are available with the paper online.

\section{References}

21. Kabsch, W. XDS. Acta Crystallogr. D Biol. Crystallogr. 66, 125-132 (2010).

22. McCoy, A. J. et al. Phaser crystallographic software. J. Appl. Crystallogr. 40, 658-674 (2007).

23. Liebschner, D. et al. Macromolecular structure determination using X-rays, neutrons and electrons: recent developments in Phenix. Acta Crystallogr. D Struct. Biol. 75, 861-877 (2019).

24. Emsley, P., Lohkamp, B., Scott, W. G. \& Cowtan, K. Features and development of Coot. Acta Crystallogr. D Biol. Crystallogr. 66, 486-501 (2010).

25. Afonine, P. V. et al. Towards automated crystallographic structure refinement with phenix.refine. Acta Crystallogr. D Biol. Crystallogr. 68, 352-367 (2012).

\section{Acknowledgements}

We are grateful to the staff at beamlines BL17U1, BL18U1 and BL19U1 at Shanghai Synchrotron Radiation Facility (SSRF, China), where data were collected. This work was supported by grants from the National Key R\&D Program of China (grant no. 2017YFC0840300 to Z.R. and 2020YFA0707500.), the Project of International Cooperation and Exchanges NSFC (grant no. 81520108019 to Z.R.), the Science and Technology Commission of Shanghai Municipality (grant no. 20431900200), the Department of Science and Technology of Guangxi Zhuang Autonomous Region (grant no. 2020AB40007) and the Natural Science Foundation of China (grant no. 31970165).

\section{Author contributions}

H.Y. and Z.R. conceived the project. Z.J., Y. Zhao, H.Y. and Z.R. designed the experiments. Z.J., Y. Zhao, H.W., Y. Zhu, C.Z., X.D., J.Y. and Xiuna Yang cloned, expressed, purified and crystallized proteins. Y. Zhao, Z.J., B.Z. and T.H. collected the diffraction data. Y. Zhao, B.Z. and X.L. solved the crystal structure. Y.S. and Y.W. performed cell-based antiviral and cytotoxicity assays. Y.D. and L.Z. performed qRT-PCR and cytotoxicity assay analysis. Y. Zhao, Z.J., Y.D., Xiaobao Yang, K.Y., X.L., L.W.G., G.X., L.Z., H.Y. and Z.R. analyzed and discussed the data. Y. Zhao, Z.J., K.Y., L.W.G., L.Z., H.Y. and Z.R. wrote the manuscript.

\section{Competing interests}

The authors declare no competing interests.

\section{Additional information}

Extended data is available for this paper at https://doi.org/10.1038/s41594-020-0440-6. Supplementary information is available for this paper at https://doi.org/10.1038/ s41594-020-0440-6.

Correspondence and requests for materials should be addressed to L.Z. or H.Y.

Peer review information Inês Chen was the primary editor on this article and managed its editorial process and peer review in collaboration with the rest of the editorial team.

Reprints and permissions information is available at www.nature.com/reprints. 
a

a
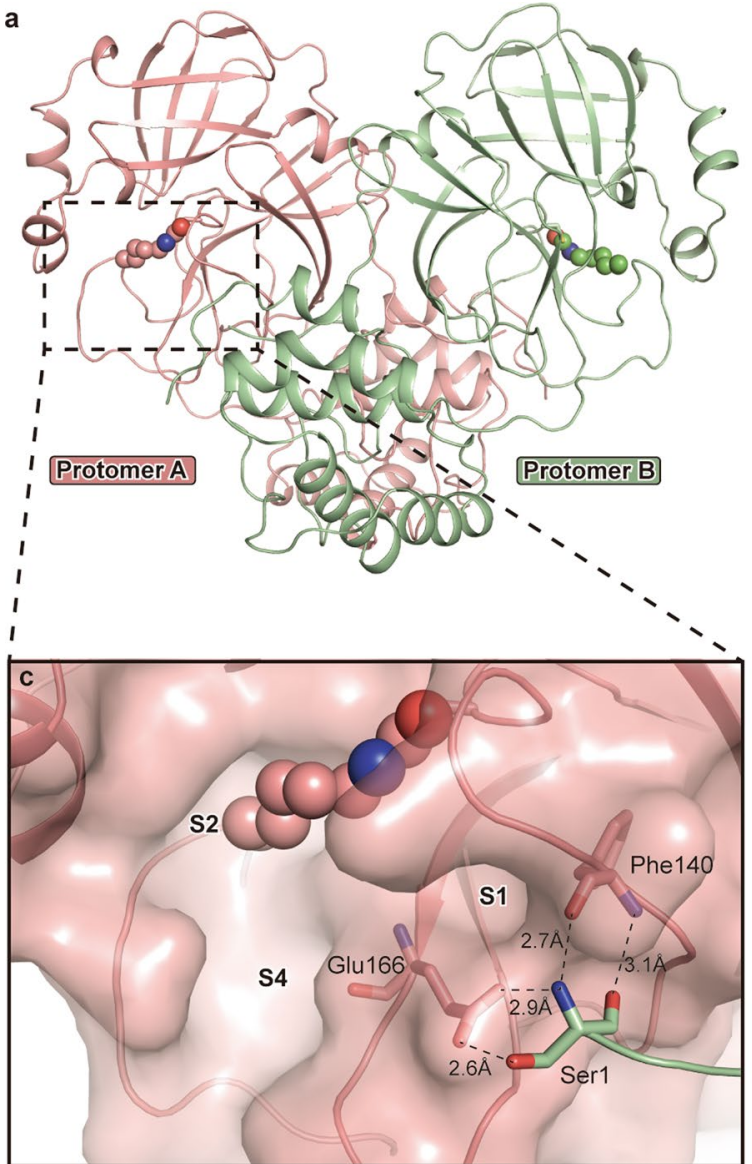

b

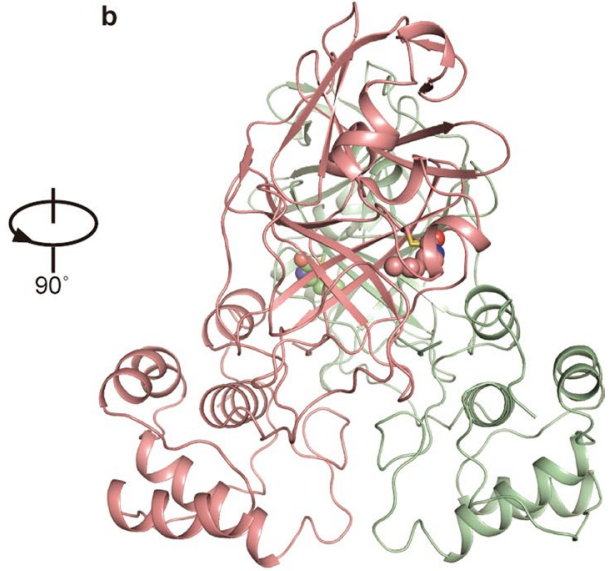

Extended Data Fig. 1 | Overall structure of SARS-CoV-2 $\mathbf{M}^{\text {pro }}$ in complex with carmofur. a, The overall structure of SARS-CoV-2 Mpro in complex with carmofur. The salmon and green represent the different protomers. The carmofur atoms are shown as solid spheres. $\mathbf{b}$, The side view of the complex. $\mathbf{c}$, The first serine participate in the formation of the dimer. 
a<smiles>CCCCCCNC(=O)N1C(=O)NC(CC)C(F)=C(F)C1=O</smiles><smiles>CCCCCCNC(=O)SCCOCC</smiles>

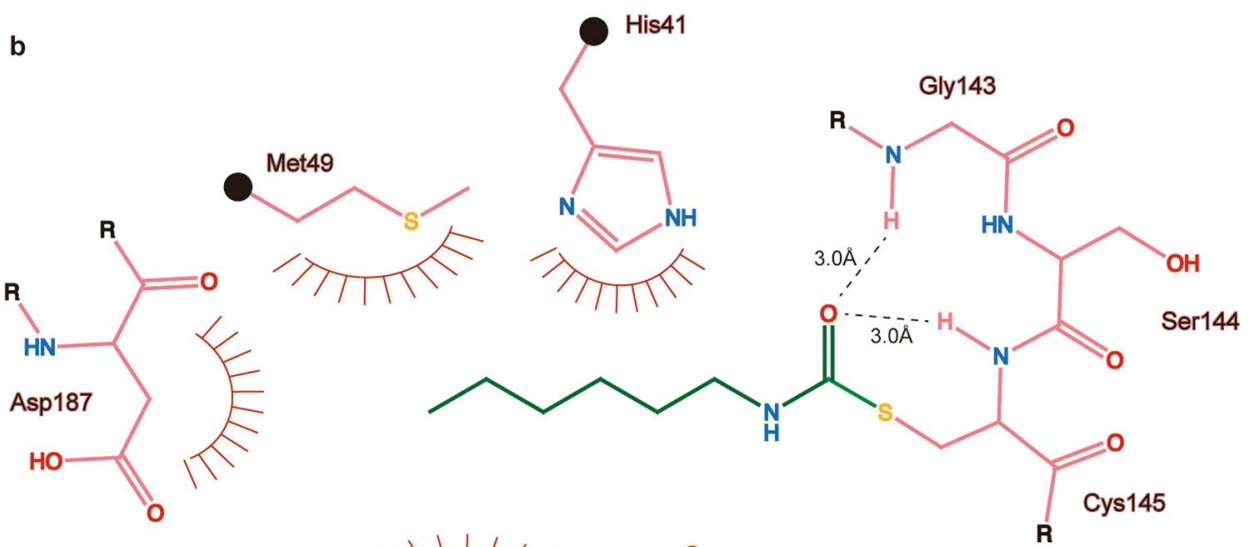<smiles>COCc1ccc(O)cc1</smiles><smiles>[R]NC(Cc1c[nH]cn1)C(=O)NC(CCSC)C([R])=O</smiles>

Extended Data Fig. 2 | Inhibition of $\mathbf{M}^{\text {pro }}$ by carmofur. a, Putative inhibition mechanism. Red curve represents $M^{\text {pro }}$ polypeptide and the black sphere

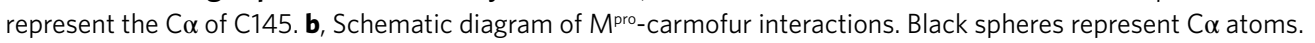




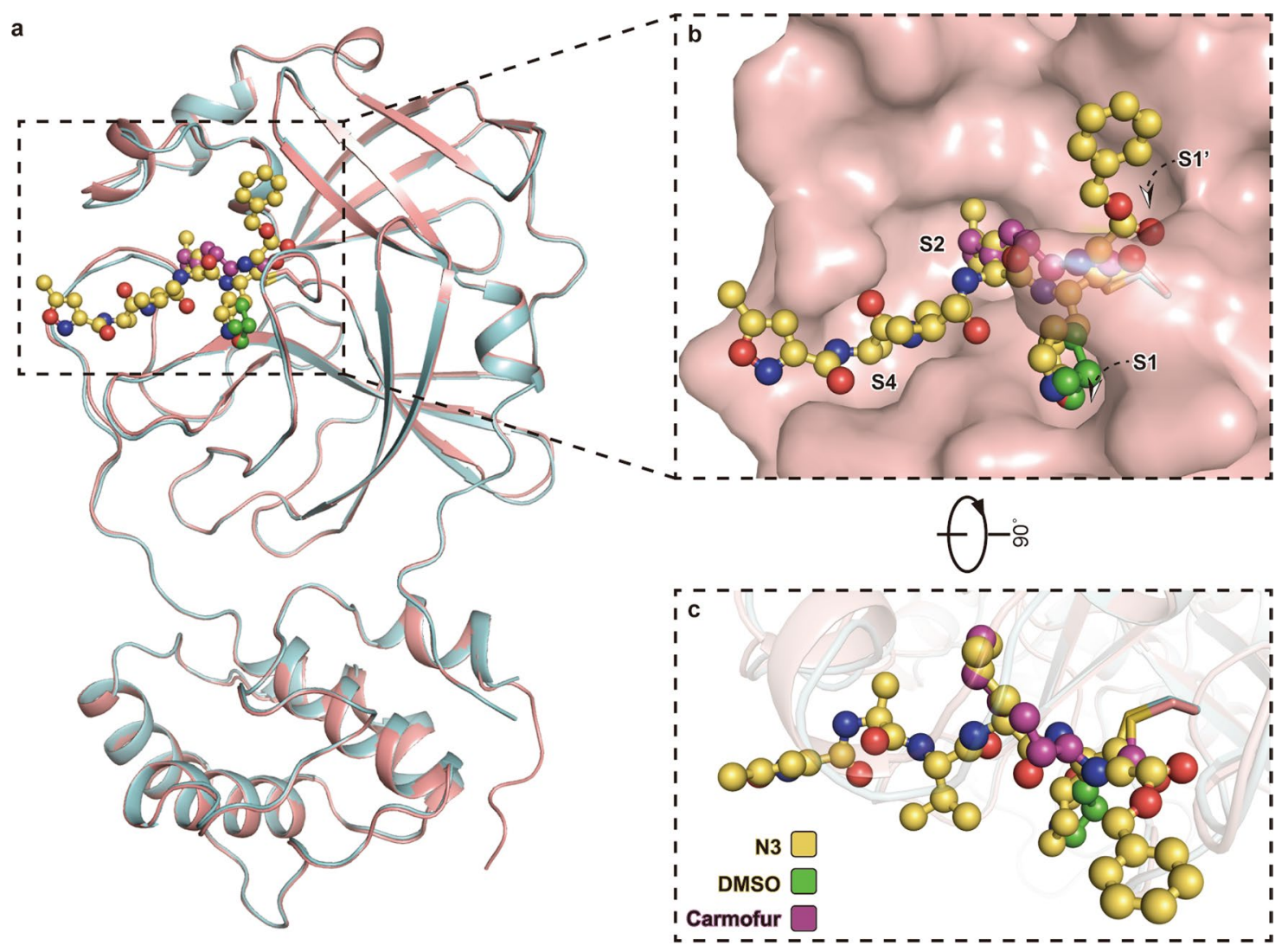

Extended Data Fig. 3 | Binding mode of carmofur and N3 to $\mathbf{M}^{\text {pro }}$. a, Overall structural comparison between the $\mathrm{M}^{\text {pro-carmofur and } M p r o-N 3}$ complexes. The salmon cartoon represents the carmofur bound structure and the light cyan represents the N3 bound structure. Carmofur, N3 and DMSO are represented by the purple, yellow and green balls and sticks, respectively. $\mathbf{b}$, The binding pocket of $\mathrm{M}^{\text {pro }}$. Carmofur and N3 are represent in the same way as in panel a. c, Schematic diagram of carmofur and N3. 


\section{Reporting Summary}

Nature Research wishes to improve the reproducibility of the work that we publish. This form provides structure for consistency and transparency in reporting. For further information on Nature Research policies, see Authors \& Referees and the Editorial Policy Checklist.

\section{Statistics}

For all statistical analyses, confirm that the following items are present in the figure legend, table legend, main text, or Methods section.

$\mathrm{n} / \mathrm{a}$ Confirmed

\ The exact sample size $(n)$ for each experimental group/condition, given as a discrete number and unit of measurement

\ A statement on whether measurements were taken from distinct samples or whether the same sample was measured repeatedly

X The statistical test(s) used AND whether they are one- or two-sided

$\triangle \square$ Only common tests should be described solely by name; describe more complex techniques in the Methods section.

Х $\square$ A description of all covariates tested

Х $\square$ A description of any assumptions or corrections, such as tests of normality and adjustment for multiple comparisons

$\triangle$ A full description of the statistical parameters including central tendency (e.g. means) or other basic estimates (e.g. regression coefficient)

AND variation (e.g. standard deviation) or associated estimates of uncertainty (e.g. confidence intervals)

$X$ For null hypothesis testing, the test statistic (e.g. $F, t, r$ ) with confidence intervals, effect sizes, degrees of freedom and $P$ value noted

Х Give $P$ values as exact values whenever suitable.

Х $\square$ For Bayesian analysis, information on the choice of priors and Markov chain Monte Carlo settings

Х $\square$ For hierarchical and complex designs, identification of the appropriate level for tests and full reporting of outcomes

Х $\square$ Estimates of effect sizes (e.g. Cohen's $d$, Pearson's $r$ ), indicating how they were calculated

\section{Our web collection on statistics for biologists contains articles on many of the points above.}

\section{Software and code}

\section{Policy information about availability of computer code}

\section{Data collection Blu-Ice BL17U1}

Data analysis $\quad$ XDS Program Package (Jan 31, 2020); PHASER (2.7.17); Phenix (1.17.1); Coot (0.8); GraphPad Prism (v8.3.1); Microsoft Excel (v16.35); PyMOL (v2.3.4)

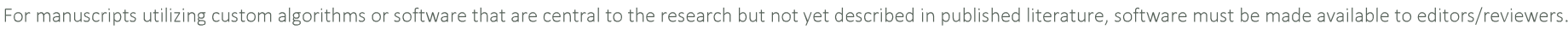
We strongly encourage code deposition in a community repository (e.g. GitHub). See the Nature Research guidelines for submitting code \& software for further information.

\section{Data}

Policy information about availability of data

All manuscripts must include a data availability statement. This statement should provide the following information, where applicable:

- Accession codes, unique identifiers, or web links for publicly available datasets

- A list of figures that have associated raw data

- A description of any restrictions on data availability

The PDB accession No. for the coordinates of COVID-19 virus Mpro in complex with Carmofur is 7BUY.

\section{Field-specific reporting}

Please select the one below that is the best fit for your research. If you are not sure, read the appropriate sections before making your selection. 


\section{Life sciences study design}

All studies must disclose on these points even when the disclosure is negative.

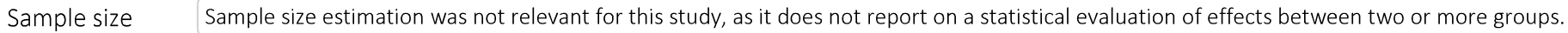

Data exclusions No data were excluded from the analysis.

Replication Antiviral (Fig 2a) and cytotoxicity (Fig 2b) assays for Carmofur were performed in six and three biological replicates respectively

Randomization Animals or human research participants were not involved in this study and, as such, samples were not randomized for the experiments.

Blinding Animals or human research participants were not involved in this study and, as such, samples were not blinded for the experiments.

\section{Reporting for specific materials, systems and methods}

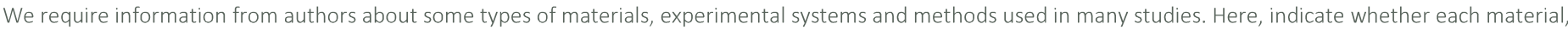

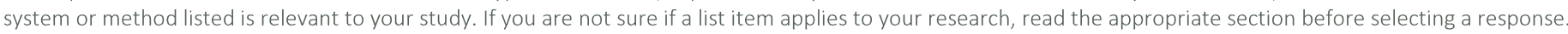

\begin{tabular}{|c|c|c|c|c|}
\hline \multicolumn{3}{|c|}{ Materials \& experimental systems } & \multicolumn{2}{|c|}{ Methods } \\
\hline $\mathrm{n} / \mathrm{a}$ & $\ln v$ & olved in the study & $\mathrm{n} / \mathrm{a}$ & Involved in the study \\
\hline Х & - & Antibodies & Х & ChIP-seq \\
\hline & Х & Eukaryotic cell lines & Х & Flow cytometry \\
\hline Х & \begin{tabular}{|c|c|c|} 
\\
\end{tabular} & Palaeontology & Х & MRI-based neuroimaging \\
\hline Х & - & Animals and other organisms & & \\
\hline Х & - & Human research participants & & \\
\hline Х & - & Clinical data & & \\
\hline
\end{tabular}

\section{Eukaryotic cell lines}

Policy information about cell lines

Cell line source(s)

Authentication

Mycoplasma contamination

Commonly misidentified lines (See ICLAC register)
African green monkey origin, Vero E6 from ATCC.

All monkey cells were from ATCC with authentication. The authentication was performed by morphology check under microscopes and growth curve analysis.

We confirm that all cells were tested as mycoplasma negative.

No commonly misidentified cell lines were used. 